\title{
Geometric Decomposition of Spider Tree
}

\author{
E. Ebin Raja Merly ${ }^{1}$, D. Subitha ${ }^{2}$ \\ ${ }^{1}$ Assistant Professor, Nesamony Memorial Christian college, Marthandam. Kanyakumari District, Tamil Nadu-629 165, India \\ ${ }^{2}$ Research Scholar, Nesamony Memorial Christian college, Marthandam. Kanyakumari District, Tamil Nadu-629 165, India.
}

\begin{abstract}
Let $G=(V, E)$ be a simple connected graph with $p$ vertices and $q$ edges. If $G_{1}, G_{2}, G_{3}, \ldots$, $G_{n}$ are connected edge disjoint subgraphs of $G$ with $E(G)=E\left(G_{1}\right) \cup E\left(G_{2}\right) \cup E\left(G_{3}\right) \cup \ldots \cup E\left(G_{n}\right)$, then $\left(G_{1}, G_{2}, G_{3}, \ldots, G_{n}\right)$ is said to be a decomposition of $G$. $A$ decomposition $\left(G_{1}, G_{2}, G_{3}, \ldots, G_{n}\right)$ of $G$ is said to be an Arithmetic Decomposition if each $G_{i}$ is connected and $\left|E\left(G_{i}\right)\right|=a+(i-1) d$, for every $i=1,2,3, \ldots, n$ and $a, d \in N$. In this paper, we introduced a new concept Geometric Decomposition. A decomposition $\left(G_{a}, G_{a r}\right.$, $\left.G_{a r}{ }^{2}, G_{a r}{ }^{3}, \ldots, G_{a r}{ }^{n-1}\right)$ of $G$ is said to be a Geometric Decomposition $(G D)$ if each $G_{a r} r^{i-1}$ is connected and $\left|E\left(G_{a r} r^{i-1}\right)\right|=a r^{i-1}$, for every $i=1$, $2,3, \ldots, n$ and $a, r \in N$. Clearly $q=\frac{a\left(r^{n}-1\right]}{\gamma-1}$. If $a=1$ and $r=2$, then $q=2^{n}-1$. In this paper we study the Geometric Decomposition of spider tree.
\end{abstract}

Keywords: Decomposition, Arithmetic Decomposition(AD), Geometric Decomposition(GD), Geometric Path Decomposition(GPD), Geometric Star Decomposition(GSD).

\section{Introduction}

In this paper, we consider simple undirected graph without loops or multiple edges. For all other standard terminology and notations we follow Harary [1].

N.Gnanadhas and J.Paulraj Joseph introduced the concept of Continuous Monotonic Decomposition (CMD) of graphs [2]. E. Ebin Raja Merly and N. Gnanadhas introduced the concept of Arithmetic Odd Decomposition (AOD) of spider tree [3].

\section{Definition: 1.1}

Let $G=(V, E)$ be a simple connected graph with $p$ vertices and $q$ edges. If $G_{1}, G_{2}, G_{3}, \ldots, G_{n}$ are connected edge disjoint subgraphs of $G$ with

$E(G)=E\left(G_{1}\right) \cup E\left(G_{2}\right) \cup E\left(G_{3}\right) \cup \ldots \cup E\left(G_{n}\right)$, then

$\left(G_{1}, G_{2}, G_{3}, \ldots, G_{n}\right)$ is said to be a decomposition of $G$.

\section{Definition: 1.2}

A decomposition $\left(G_{1}, G_{2}, G_{3}, \ldots, G_{n}\right)$ of $G$ is said to be an Arithmetic Decomposition(AD) if each $G_{i}$ is connected and $\left|E\left(G_{i}\right)\right|=a+(i-1) d$, for every $i=1,2,3, \ldots, n$ and $a, d \in \mathrm{N}$.

\section{Definition: 1.3}

Let $G$ be a connected graph. The diameter of $G$ is defined as $\max \{\mathrm{d}(u, v): u, v \in V(G)\}$ and is denoted by $\operatorname{diam}(G)$.

\section{Geometric Decomposition of Graphs}

\section{Definition: 2.1}

A decomposition $\left(G_{a}, G_{a r}, G_{a r}^{2}, G_{a r^{3}}, \ldots, G_{a r}{ }^{n-1}\right)$ of $G$ is said to be a Geometric Decomposition(GD) if each $G_{a r}{ }^{i-1}$ is connected and $\left|E\left(G_{a r} r^{i-1}\right)\right|=a r^{i-1}$, for every $i=1,2,3, \ldots, n$ and $a, r \in \mathrm{N}$. Clearly $q=\frac{\bar{a}\left(r^{2}-1\right)}{\gamma-1}$. If $a=1$ and $r=2$, then $q$ $=2^{n}-1$.
We know that $2^{n-1}$ is the sum of $2^{0}, 2^{1}, 2^{2}, 2^{3}, \ldots, 2^{n-1}$. That is, $2^{n}-1$ is the sum of $1,2,4,8, \ldots, 2^{n-1}$. Thus we denote the GD as $\left(G_{1}, G_{2}, G_{4}, \ldots, G_{2}{ }^{n-1}\right)$.

Example: 2.2

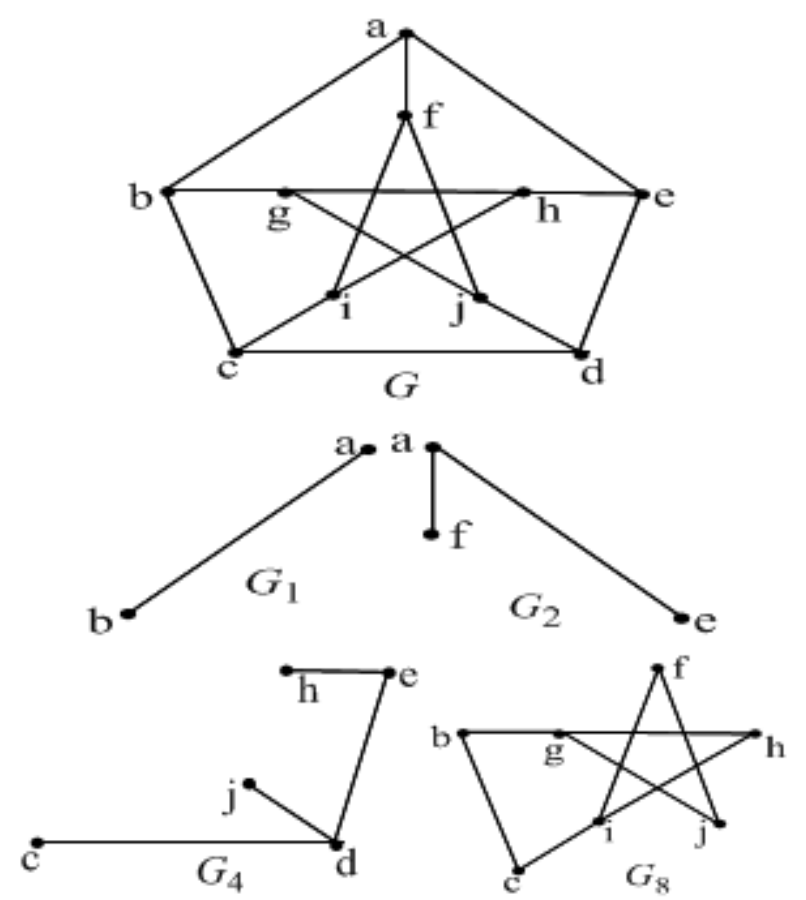

Figure 1: A Petersen graph admits GD $\left(G_{1}, G_{2}, G_{4}, G_{8}\right)$ of G.

Theorem 2.3: A graph $G$ admits GD $\left(G_{1}, G_{2}, G_{4}, \ldots, G_{2}^{n-1}\right)$ if and only if $q=2^{n}-1$ for each $n \in \mathrm{N}$

Proof:

Let $G$ be a connected graph with $q=2^{n}-1$. Let $u, v$ be two vertices of $G$ such that $d(u, v)$ is maximum. Let $N_{r}(u)=$ $\{v \in V / d(u, v)=r\}$. If $d(u)=2^{n-1}$, choose $2^{n-1}$ edges incident with $u$. Let $G_{2}^{n-1}$ be a subgraph induced by these $2^{n-}$ 1 edges. If $d(u)<2^{n-1}$, then choose $2^{n-1}$ edges incident with 


\section{International Journal of Science and Research (IJSR) \\ ISSN (Online): 2319-7064}

Index Copernicus Value (2013): 6.14 | Impact Factor (2014): 5.611

$u$ vertices of $N_{1}(u), N_{2}(u),{ }^{m x}$ successively such that the subgraph $G_{2}{ }^{n-1}$ induced by these edges is connected. In both cases $G-G_{2^{n-1}}$ has a connected component $H_{1}$ with $2^{n}-2^{n-1}$ - 1 edges.

Now, consider $H_{1}$ and proceed as above to get $G_{2}{ }^{n-2}$ such that $H_{1}-G_{2}{ }^{n-2}$ has a connected component $H_{2}$ of size $2^{n}-2^{n-1}$ $2^{n-2}-1$ edges. Proceeding like this we get a connected subgraph $G_{2}$ such that $H_{2}{ }^{n-2}$ is a graph with one edge taken as $G_{1}$. Thus $\left(G_{1}, G_{2}, G_{4}, \ldots, G_{2^{n-1}}\right)$ is a GD of $G$.

Conversely, Suppose $G$ admits GD $\left(G_{1}, G_{2}, G_{4}, \ldots, G_{2^{n-1}}\right)$. Then obviously, $q(G)=1+2+4+\ldots+2^{n-1}=2^{n}-1$ for each $n \in \mathrm{N}$.

\section{Definition 2.4:}

A GD in which each $G_{2} 2^{\mathrm{i}-1}$ is a path of size $2^{\mathrm{i}-1}$ is said to be a Geometric Path Decomposition (GPD).

\section{Example 2.5:}
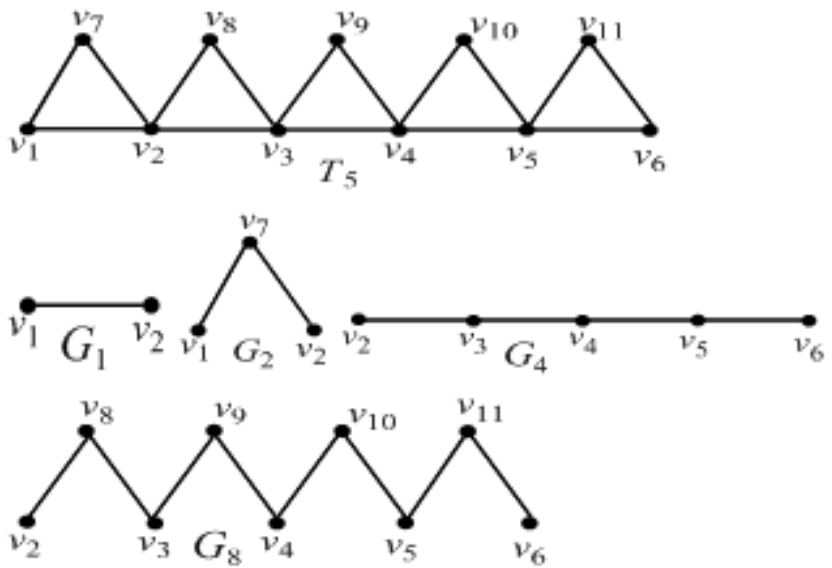

Figure 2: A triangular snake graph $T_{5}$ admits GPD.

\section{Definition: 2.6}

A GD in which each $G_{2} 2^{\mathrm{i}-1}$ is a star of size $2^{\mathrm{i}-1}$ is said to be a Geometric star Decomposition (GSD).

\section{Example: 2.7}
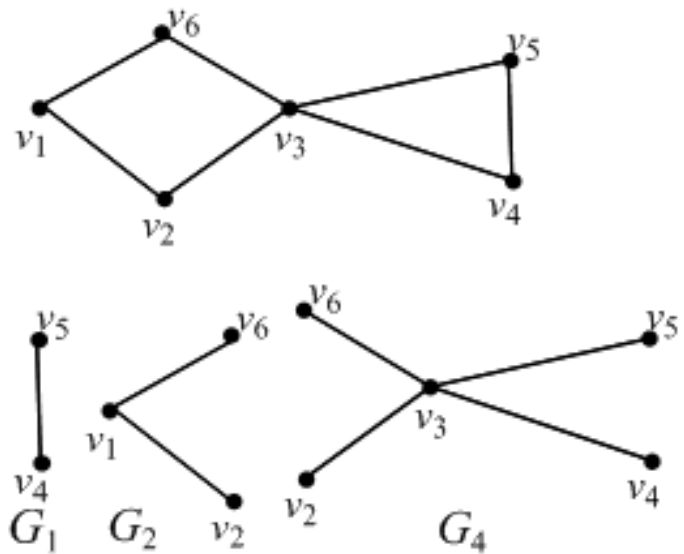

Figure 3: Fish graph admits GSD.

\section{Geometric Decomposition of Spider Graphs}

Definition 3.1: A tree $\mathrm{T}$ with exactly one vertex of degree $\geq$ 3 is called a Spider tree.

Notation 3.2: Let $W$ denote the set of pendent vertices of $T$ and $\mathrm{u}$ be the vertex of degree $\geq 3$ in $\mathrm{T}$.

Theorem 3.3 : If $\mathrm{T}$ is a spider tree with $\operatorname{diam}(\mathrm{T})=\mathrm{t}, 2 \leq$ $\mathrm{t} \leq 5$ with $\mathrm{d}(u)=\left(2^{n}-1\right)-(\mathrm{t}-2)$, then $\mathrm{T}$ admits GSD.

Proof:

Case (i): $t=2$. Since diam $(T)=2, T$ is a star. Also, since $\mathrm{d}(u)=2^{n}-1$, $\mathrm{T}$ is $\mathrm{K}_{1,2^{n}-1}$. Therefore, $q(\mathrm{~T})=2^{n}-1$. Hence $\mathrm{T}$ admits GSD.

Case (ii) $\mathrm{t}=3$. Since $\operatorname{diam}(\mathrm{T})=3$ and $d(u)=\left(2^{n}-1\right)-1$, there are $\left(2^{n}-1\right)-2$ pendent edges incident with $u$. Let $S_{1}=$ $e$. Then T- $e$ is a star $K_{1,\left(2^{n}-1\right)-1}$ and $q(\mathrm{~T}-e)=\left(2^{n}-1\right)-1$. Then we can easily decompose T - $e$ into $S_{2}, S_{4}, S_{8}, \ldots, S_{2^{n-1}}$. Hence $\mathrm{T}$ admits GSD.

Case (iii) $\mathrm{t}=4$.

Subcase (i): $u$ is the origin of $P_{3}$.

Let $u_{1}$ be a non pendent vertex adjacent to $u$ and $u_{2}$ be a terminus of $u-u_{2}$ path of length 3 . Let $S_{1}=u_{1} u$ and $S_{2}=u_{2}$ $u_{1}$. Then the remaining edges of tree is a star which can be decomposed into $S_{4}, S_{8}, S_{16}, \ldots, S_{2}{ }^{n-1}$

Subcase(ii): $u$ is not the origin of $P_{3}$.

Let $u_{1}$ and $u_{2}$ be the two non pendent vertices adjacent to $u$ and let $v_{1}$ and $v_{2}$ be the pendent vertices adjacent to $u_{1}$ and $u_{2}$ respectively. Then $S_{1}=u_{1} v_{1}$ and $S_{2}=u-v_{2}$ path in T and the remaining edges form a star $K_{1,\left(2^{n}-1\right)-3}$. Then we can easily decomposed into $S_{4}, S_{8}, S_{16}, \ldots, S_{2^{n-1}}$

Case (iv) $\mathrm{t}=5$.

Subcase (i): $u$ is the origin of $P_{4}$.

Let $u_{1}$ be a non pendent vertex adjacent to $u$ and $u_{2}$ be a terminus of $u-u_{2}$ path of length 4 . Then $u_{2}-u_{1}$ path can be decomposed in to $S_{1}, S_{2}$ and the remaining edges is a star. Clearly $q\left(\mathrm{~T}-\left\{S_{1} S_{2}\right\}\right)=\left(2^{n}-1\right)-3$. Then T can easily decompose $S_{4}, S_{8}, S_{16}, \ldots, S_{2}^{n-1}$.

Subcase(ii): $u$ is not the origin of $P_{4}$.

Let $u_{1}$ and $u_{2}$ be the two non pendent vertices adjacent to $u$ and $v_{1}$ be a pendent vertex adjacent to $u_{1}$. Let $v_{2}$ be the pendent vertex of $\mathrm{T}$ such that there is a $u_{2}-v_{2}$ path of length 2 is adjacent to $u_{2}$. Then $S_{1}=u_{1} v_{1}$ and $S_{2}=u_{2}-v_{2}$ path in $\mathrm{T}$ and the remaining edges is a star with $\left(2^{n}-1\right)-3$ edges. Hence T admits GSD

Theorem 3.4 : If $\mathrm{T}$ is a spider tree with $\operatorname{diam}(\mathrm{T})=\mathrm{t}, 3 \leq$ $\mathrm{t} \leq 5$ and $\mathrm{d}(u)=\left(2^{n}-1\right)-(\mathrm{t}-2)$ admits GSD if and only if $\mathrm{T}$ - $\mathrm{W}=P_{x}$ where $x \leq 3$.

Proof:

Assume T $-\mathrm{W}=P_{x}$ where $x \leq 3$. Then by previous theorem $\mathrm{T}$ admits GSD. Conversely, the result is obvious. 


\section{International Journal of Science and Research (IJSR) \\ ISSN (Online): 2319-7064 \\ Index Copernicus Value (2013): 6.14 | Impact Factor (2014): 5.611}

Result 3.5: If $\mathrm{T}$ is a spider tree with $\operatorname{diam}(\mathrm{T})=2$ and $\mathrm{d}(u)=$ 3 , then $\mathrm{T}$ admits GSD and GPD.

Proof:

Since $\operatorname{diam}(\mathrm{T})=2$ and $\mathrm{d}(u)=3$. Clearly $\mathrm{T}$ is a spider tree with 3 edges. Then we can easily decompose $\mathrm{T}$ into paths $P_{1}$ and $P_{2}$. Therefore, by theorem(3.3) T admits GSD and GPD.

Result 3.6: If $\mathrm{T}$ is a spider tree with $\operatorname{diam}(\mathrm{T})=4$ and $\mathrm{d}(u)=$ 5, then T admits GSD and GPD.

Proof:

Since $\operatorname{diam}(\mathrm{T})=4$ and $\mathrm{d}(u)=5$, then there is a path of length 4. Therefore, the spider tree can be decomposed into $P_{1}, P_{2}$ and $P_{4}$. Also by theorem (3.3) $\mathrm{T}$ admits GSD and GPD.

Result 3.7: If $\mathrm{T}$ is a spider tree with $\operatorname{diam}(\mathrm{T})=5$ and $\mathrm{d}(u)=$ 4,then T admits GSD and GPD.

\section{Proof:}

Sinc Since $\operatorname{diam}(\mathrm{T})=5$, then there is path of length 5 . Then $P_{5}$ can be decomposed into $P_{1}$ and $P_{4}$. Also by theorem (3.3) T admits GPD and GSD.

\section{Results 3.8:}

(i) If $\mathrm{T}$ is a spider tree with $\left(2^{n}-1\right)-5 \leq \operatorname{diam}(\mathrm{T}) \leq\left(2^{n}\right.$. 1) - 1 , then $T$ admits GPD but not GSD.

(ii) If $\mathrm{T}$ is a spider tree with $6 \leq \operatorname{diam}(\mathrm{T}) \leq\left(2^{n}-1\right)-6$, then $\mathrm{T}$ admits neither GPD nor GSD.

Example 3.9: Consider a spider tree $\mathrm{T}$ with $q=15$.

\begin{tabular}{|c|c|c|}
\hline Diam $(\mathrm{T})$ & GSD & GPD \\
\hline 2 & Yes & No \\
\hline 3 & Yes & No \\
\hline 4 & Yes & No \\
\hline 5 & Yes & No \\
\hline 6 & No & No \\
\hline 7 & No & No \\
\hline 8 & No & No \\
\hline 9 & No & No \\
\hline 10 & No & Yes \\
\hline 11 & No & Yes \\
\hline 12 & No & Yes \\
\hline 13 & No & Yes \\
\hline 14 & No & Yes \\
\hline
\end{tabular}

\section{References}

[1] Harary,F.,1988, "Graph Theory",Narosa Publishing House, New Delhi.

[2] Gnanadhas, N., and Paulrai Joseph, J.,2000 "Continuous Monotonic Decomposition of Graphs", International Journal of Management and Systems.,Vol.16,pp.333 344.

[3] Ebin Raja Merly, E., and Gnanadhas, N., 2011 "Arithmetic Odd Decomposition of Spider tree" Asian Journal of Current Engineering and Maths., 2:2 MarchApril (2013) pp.99-101.

[4] Foulds, L. R., 1991, "Graph Theory Application", Narosa Publishing House, New Delhi.

[5] Nagarajan, A., and Navavaneetha Krishnan, S., 2005"Continuous Monotonic Decomposition of some special class of Graphs" ,International Journal of Management and Systems.,Vol.21,No.1, pp.91 - 106. 